Review began 02/01/2022 Review ended 02/04/2022 Published 02/13/2022

(c) Copyright 2022

AlYami et al. This is an open access article distributed under the terms of the Creative Commons Attribution License CC-BY 4.0., which permits unrestricted use, distribution, and reproduction in any medium, provided the original author and source are credited

\section{Association Between Osteoporosis and Refracture Rate Among Patients With Hip Fractures at King Abdulaziz Medical City, Saudi Arabia}

Ali H. AlYami 1, 2 , Majed N. Alosaimi 3, 4, 5 , Mohammed S. Alshehri 3, 4, 5 , Abdulhamid T. Alghamdi 5, 6 Majd A. SaemAldahar ${ }^{7,6}$, Turki A. Alsafrani ${ }^{7,6}$, Albaraa A. Dabroom ${ }^{7,6}$, Ibrahim A. Kattan ${ }^{7,6}$, Fares M. Sindi 7, 6 , Azzam M. Azaya 7, 6 , Bandar N. AlMaeen 8, 9

\begin{abstract}
1. Department of Surgery, Ministry of National Guard Health Affairs, Jeddah, SAU 2. Department of Surgery, King Saud Bin Abdulaziz University for Health Sciences, Jeddah, SAU 3. Department of Orthopaedic Surgery, Ministry of National Guard Health Affairs, Jeddah, SAU 4. Department of Orthopaedics, King Abdullah International Medical Research Center, Riyadh, SAU 5. Department of Orthopaedics, King Saud Bin Abdulaziz University for Health Sciences, Jeddah, SAU 6. College of Medicine, Ministry of National Guard Health Affairs, Jeddah, SAU 7. College of Medicine, King Saud Bin Abdulaziz University for Health Sciences, Jeddah, SAU 8. Department of Surgery, College of Medicine, Jouf University, Al-Jouf, SAU 9. Department of Orthopaedic Surgery, King Faisal Specialist Hospital and Research Centre, Riyadh, SAU
\end{abstract}

Corresponding author: Bandar N. AlMaeen, d-bandar@hotmail.com

\section{Abstract}

\section{Background}

Hip fracture is a major medical and surgical topic and is a significant cause of morbidity and mortality. Older women, especially those with osteoporosis, are at an increased risk for hip fractures. Multiple studies have shown the effect of osteoporosis on the refracture rate among the elderly population. Therefore, selecting a targeted population for screening and treating osteoporosis has an essential role in decreasing the hip fracture rate. This study aimed to determine the association between osteoporosis treatment and refracture rate among patients with hip fractures at King Abdulaziz Medical City, Jeddah, Saudi Arabia.

\section{Methods}

Collected data included patient demographics (men: $\geqslant 55$ years old; women: $\geqslant 50$ years old), the used osteoporosis investigation method, osteoporosis treatment history, presence of comorbidities, and refracture as a primary outcome. The refracture rate among patients with hip fracture was calculated and used to determine the association between hip refracture and osteoporosis.

\section{Results}

Our study included a total of 292 patients who presented to our hospital due to hip fractures. The patients were divided into two groups, the osteoporotic and non-osteoporotic groups. These groups were then compared. There was no statistical significance between osteoporosis and hip refracture $(\mathrm{p}=0.721)$, and there was no association between the treatment of osteoporosis and hip refracture $(\mathrm{p}=0.493)$. Statistical difference was found between patients who had undergone dual-energy X-ray absorptiometry scan and were not treated for osteoporosis $(p=0.00)$. Lastly, the mortality of the refracture group was $10 \%$, while it was $11 \%$ in the no-refracture group $(\mathrm{p}=1.00)$.

\section{Conclusion}

Morbidity and mortality rates are higher among patients with hip fractures. Our study showed that there was no association between hip refracture rate and osteoporosis whether the patient is treated for osteoporosis or not. We recommend a systematic review that can include more studies in this field to acquire more definitive results regarding this topic.

Categories: Orthopedics, Epidemiology/Public Health, Trauma

Keywords: fracture, hip, dexa, refracture, osteoporosis

\section{Introduction}

Hip fracture is a major health problem, especially in the elderly population, and is a significant cause of mortality and morbidity. The incidence of hip fracture in the United States is 80 per 100,000, and the incidence doubles each decade in patients over 50 years old. The prevalence of hip fracture is two to three times higher in women than men [1]. The mortality rate following hip fracture was reported to range between $14 \%$ and $37 \%$ within 12 months in different studies [2-4]. Different risk factors are related to hip fracture, such as decreased bone mineral density (BMD), decreased visual acuity, neuromuscular impairment, and cognitive impairment [5]. 
Osteoporosis is a skeletal disorder characterized by reduced bone strength leading to an increased risk for fracture. In this disorder, bone quality is compromised, bone strength is weakened, and most importantly, patients with osteoporosis are at an increased risk for fracture and refracture. Several studies worldwide demonstrated that there is a higher rate of refracture following an osteoporotic fracture, and the rate is approximately two to nine times higher among the elderly population. As shown in different studies worldwide, failure to adhere to therapy was the leading cause of refracture [6,7]. Osteoporosis-related fractures may have potentially catastrophic consequences, including disability and death [8]. Osteoporosis affects approximately 200 million people worldwide [9]. Screening for osteoporosis is ideally initiated in all women aged $\geqslant 65$ years old by measuring BMD of the hip and lumbar spine using dual-energy X-ray absorptiometry (DEXA).

Despite the current recommendation suggested by the United States Preventive Services Task Force (USPSTF) in osteoporosis screening, recent studies show that only $25 \%$ of women aged $65-85$ are screened for osteoporosis [10]. Furthermore, DEXA scan results are inadequately followed in treating patients undergoing osteoporosis screening. For example, one study showed that $33 \%$ of patients who underwent DEXA did not receive treatment for abnormal results, and most did not have their results reviewed [11].

Multiple studies in different countries with variable results reported the importance of treating osteoporosis and its association with reducing hip refracture rate and mortality $[12,13,14]$. However, local data on this topic are lacking. Thus, this study aimed to determine the association between osteoporosis and refracture rate among patients with hip fractures at King Abdulaziz Medical City, Jeddah, Saudi Arabia.

\section{Materials And Methods}

This retrospective cohort study included all patients diagnosed with hip fracture, fractures in the femoral head, neck of femur, and intertrochanteric and per-trochanteric fractures. Male patients $\geqslant 55$ years old and female patients $\$ 50$ years old were included in this study at King Abdulaziz Medical City in Jeddah, Saudi Arabia. Data were gathered using a data collection sheet to collect patient demographics, such as age, gender, weight, and height, the used osteoporosis investigation method, osteoporosis treatment history, the presence of co-morbidities, and the outcome. All data were acquired from Best Care System using electronic medical records at King Abdulaziz Medical City. The refracture rate among patients with hip fracture was calculated and used to determine the association between hip refracture and osteoporosis as a primary objective. A DEXA scan was used as the method for screening and diagnosing osteoporosis. Primary treatments for osteoporosis included denosumab, teriparatide, and alendronate. Calcium and vitamin D supplements were not considered as primary treatments for osteoporosis. Our primary outcome was hip refracture, including the type of refracture and the occurrence of death from refracture.

The sample size was calculated using ClinCalc.com by setting the lifetime incidence of hip fracture to 15$18 \%$. Moreover, the anticipated fracture rate in our study is $30 \%$, with a margin of error of $5 \%$, and the power of the study is $80 \%$. The total sample size was 630 , with 315 in each group. However, a non-probability consecutive sampling technique was used to include all patients who met the inclusion and exclusion criteria. Data were collected by the co-authors of the study. Ethical approval was obtained from the institutional review board, and scientific approval was obtained from King Abdullah International Medical Research Center.

IBM SPSS version 23 was used for data analysis. Categorical variables were reported as percentages, and numerical variables were reported as means or medians. Normally distributed numerical variables were reported using the mean and SD, and skewed distributed variables were reported using median and interquartile range (IQR). Chi-squared test and Fisher's exact test were used to analyze the association between osteoporosis and refracture rate, and it was also used in other variables, such as the DEXA scan results, to analyze their relation to patients treated and not treated for osteoporosis. Statistical significance was set at a p-value of $<0.05$.

\section{Results}

We conducted a retrospective cohort study that included a total of 292 patients who have been subjected to hip fracture in our hospital. The median age in the osteoporotic group is 74 years, while in the nonosteoporotic group is 73 years. Most patients were females in the osteoporotic group (66.7\%) and males in the non-osteoporotic group (58.5\%). The most common fracture was femoral neck fracture (50\% in females and $49.5 \%$ in males). Diabetes mellitus and hypertension were prevalent in both groups ( $50.7 \%$ and $47.9 \%$ in females; $72 \%$ and $58.5 \%$ in males, respectively). Other demographics and co-morbidities are summarized in Table 1. 


\section{Cureus}

\begin{tabular}{|c|c|c|c|c|}
\hline \multicolumn{2}{|l|}{ Demographics } & Osteoporotic & Non-osteoporotic & $p$-value \\
\hline \multirow{2}{*}{ Sex } & Male & $25(33.3 \%)$ & $127(58.5 \%)$ & \multirow{2}{*}{$0.00^{*}$} \\
\hline & Female & $50(66.7 \%)$ & $90(41.5 \%)$ & \\
\hline \multirow{2}{*}{ Calcium/Vitamin D supplements } & Yes & $55(73.3 \%)$ & $78(35.9 \%)$ & \multirow{2}{*}{$0.00^{*}$} \\
\hline & No & $20(26.7 \%)$ & 139 (64.1\%) & \\
\hline \multirow{5}{*}{ Type of fracture of the first fracture } & Acetabular fracture & $1(1.4 \%)$ & $9(4.2 \%)$ & \multirow{5}{*}{$0.71^{*}$} \\
\hline & Femoral neck fracture & $37(50 \%)$ & 106 (49.5\%) & \\
\hline & Intertrochanteric Fracture & $35(47.3 \%)$ & $96(44.9 \%)$ & \\
\hline & Subtrochanteric fracture & $1(1.4 \%)$ & $3(1.4 \%)$ & \\
\hline & Total fractures & $74(100 \%)$ & $214(100 \%)$ & \\
\hline \multirow{2}{*}{ Diabetes mellitus } & Yes & $38(50.7 \%)$ & $104(47.9 \%)$ & \multirow{2}{*}{$0.68^{*}$} \\
\hline & No & $37(49.3 \%)$ & $113(52.1 \%)$ & \\
\hline Hypertension & Yes & $54(72 \%)$ & $127(58.5 \%)$ & $0.038^{\star}$ \\
\hline \multirow{2}{*}{ Dyslipidemia } & Yes & $19(25.3 \%)$ & $36(16.6 \%)$ & \multirow{2}{*}{$0.095^{*}$} \\
\hline & No & $56(74.7 \%)$ & $181(83.4 \%)$ & \\
\hline \multirow{2}{*}{ Congestive heart failure } & Yes & $12(16 \%)$ & $19(8.8 \%)$ & \multirow{2}{*}{$0.079^{*}$} \\
\hline & No & $63(84 \%)$ & $198(91.2 \%)$ & \\
\hline \multirow{2}{*}{ Arthritis } & Yes & $16(21.3 \%)$ & $15(6.9 \%)$ & \multirow{2}{*}{$0.00^{*}$} \\
\hline & No & $59(78.7 \%)$ & 202 (93.1\%) & \\
\hline \multirow{2}{*}{ Chronic kidney disease } & Yes & $15(20 \%)$ & $35(16.1 \%)$ & \multirow{2}{*}{$0.44^{*}$} \\
\hline & No & $60(80 \%)$ & $182(83.9 \%)$ & \\
\hline \multirow{2}{*}{ Hypothyroidism } & Yes & $6(8 \%)$ & $12(5.5 \%)$ & \multirow{2}{*}{$0.41^{* *}$} \\
\hline & No & $69(92 \%)$ & 205 (94.5\%) & \\
\hline \multirow{2}{*}{ Hyperparathyroidism } & Yes & $1(1.3 \%)$ & $2(0.9 \%)$ & \multirow{2}{*}{$1.00^{* *}$} \\
\hline & No & $74(98.7 \%)$ & 215 (99.1\%) & \\
\hline \multirow{2}{*}{ Cancer/Tumor } & Yes & $8(10.7 \%)$ & $25(11.5 \%)$ & \multirow{2}{*}{$0.84^{*}$} \\
\hline & No & $67(89.3 \%)$ & $192(88.5 \%)$ & \\
\hline \multicolumn{2}{|l|}{ Age, Median (IQR) } & $74(18)$ & $73(25)$ & \\
\hline \multicolumn{2}{|l|}{ BMI, Median (IQR) } & $26.6(8.4)$ & $25.4(7.5)$ & \\
\hline
\end{tabular}

\section{TABLE 1: General demographics.}

*: Chi-squared test; **: Fisher's exact test; IQR: Interquartile range.

Table 2 demonstrates the primary objective outcome comparing the refracture in both groups with osteoporosis. Most patients in the refracture and no-refracture groups were non-osteoporotic ( $70 \%$ and $74.5 \%$, respectively). No statistical significance was found using Fisher's exact test between both groups ( $\mathrm{p}=$ 0.721). We compared the refracture rate with treatment effect to further assess the impact of the treatment. Most patients in the refracture and no-refracture groups did not receive treatment. Fisher's exact test showed no statistical significance between the refracture group and osteoporosis treatment $(p=0.493)$. 


\section{Cureus}

\begin{tabular}{|c|c|c|c|c|}
\hline Outcome & & Refracture & No refracture & p-value \\
\hline \multirow{2}{*}{ Osteoporosis } & Yes & $3(30 \%)$ & $72(25.5 \%)$ & \multirow{2}{*}{$0.721^{* *}$} \\
\hline & No & $7(70 \%)$ & $210(74.5 \%)$ & \\
\hline \multirow{2}{*}{ Treated osteoporosis (in osteoporotic group) } & Yes & $1(33.3 \%)$ & $14(19.4 \%)$ & \multirow{2}{*}{$0.493^{* *}$} \\
\hline & No & $2(66.7 \%)$ & 58 (80.6\%) & \\
\hline
\end{tabular}

TABLE 2: Primary objective outcome comparing the refracture in both groups with osteoporosis.

**: Fisher's exact test.

To assess the investigation across the sample, we compared the patients who underwent a DEXA scan and started to receive treatment with those who did not. Seventy-eight patients had done the scan, yet only 14 out of those 78 received treatment. Statistical significance was found between the groups $(\mathrm{p}=0.00)$. The distribution of both groups is shown in Table 3.

\begin{tabular}{|c|c|c|c|c|}
\hline \multicolumn{2}{|l|}{ Factor } & Treated for osteoporosis & Not treated for osteoporosis & $\mathrm{p}$-value \\
\hline \multirow{2}{*}{ DEXA scan } & Yes & $14(17.9 \%)$ & $64(82.1 \%)$ & \multirow{2}{*}{$0.00^{\star \star}$} \\
\hline & No & $3(1.4 \%)$ & $211(98.6 \%)$ & \\
\hline
\end{tabular}

\section{TABLE 3: Investigation outcome.}

**: Fisher's exact test.

The mortality of the refracture group is $10 \%(1 / 10)$, while that of the no-refracture group is $11 \%$. Table 4 shows no statistical significance between mortality and refracture $(\mathrm{p}=1.00)$.

\begin{tabular}{|c|c|c|c|c|}
\hline Outcome & & Died & Did not die & p-value \\
\hline \multirow{2}{*}{ Refracture } & Yes & $1(10 \%)$ & $9(90 \%)$ & \multirow{2}{*}{$1.00^{\star \star}$} \\
\hline & No & $31(11 \%)$ & 251 (89\%) & \\
\hline
\end{tabular}

\section{TABLE 4: Mortality in the refracture groups.}

**: Fisher's exact test.

\section{Discussion}

Our analysis showed that in patients with a history of a hip fracture, 26.71\% were screened for osteoporosis using a DEXA scan. This percentage is comparable to the numbers reported in similar studies. According to Murray AW et al., the population in their study involved two centers. Patients who were offered or already had undergone a DEXA scan were $19.4 \%$ in one center and $40.5 \%$ in another center [15]. Similarly, in an interventional study by Davis JC et al., 29\% of patients had received a DEXA scan after a hip fracture. This number was significantly increased compared to their control population [16].

Since populations who suffer from osteoporosis are usually elderly, they have a higher possibility of having other co-morbidities; therefore, it is essential to discuss the possible association of osteoporosis with different factors. For instance, there was no significant association between type 2 diabetes mellitus and osteoporosis in our study. Saller A et al. reviewed multiple articles regarding this association, which reported contradicting findings; some studies reported a higher BMD in patients who had diabetes, while other studies noticed sex-related differences in BMD changes [17]. Data remain unclear regarding the association between these diseases. However, it is essential to consider other factors that contribute to fractures. For example, visual impairment and peripheral neuropathy associated with type 2 diabetes may precipitate falls, 
Our study reported a significant statistical association between arthritis and osteoporosis. Patients with osteoporosis were less likely to have arthritis; however, studies by Geusens PP et al. and Bultink IE et al. have reported their coexistence. They were considered to coexist due to factors related to body composition and the underlying inflammatory processes. In addition, there are other similar demographic factors, such as age and gender $[18,19]$.

Of the 75 patients diagnosed with osteoporosis, only $20 \%$ were treated for osteoporosis. This number is low compared to the study by Satomi et al., in which $43 \%$ of the patients with previously diagnosed osteoporosis have received treatment [19]. This difference is mainly attributed to the definition of the treatment of osteoporosis. In the study by Satomi E et al., supplementation with calcium only was considered a treatment; however, it was not in our study. Let's consider these modalities to be a proper treatment. The percentage of treated osteoporotic patients will increase to $41.35 \%$, comparable to multiple other studies, such as those by Satomi E et al. and Smith MD et al. [20, 21].

In measuring the outcome of patients, we investigated the incidence of refracture and mortality rate. Although patients with a history of hip fracture who are osteoporotic are expected to have a higher rate of refracture, our results did not show this. Only $4.44 \%$ of the osteoporotic group experienced a refracture. In contrast, the study by Bliuc D et al., which only included osteoporotic patients, reported a refracture rate of $34.59 \%$ [22]. Furthermore, Ryg J et al. showed that the hip refracture rate was 11-23\% [23]. However, this discrepancy may be because only a quarter of our study population had osteoporotic screening and other fracture risk assessments after their initial fracture. In addition, most studies investigating refractures also included non-hip fractures, while our study excluded them. Moreover, longer follow-up periods may predispose patients to a higher number of refracture incidences, which was included in the study by Ryg J et al. [23]. Misclassification of other complications, such as infections, periprosthetic fractures, and hip refractures, may overestimate and exaggerate the actual percentages.

The other measured outcome was the mortality rate. Based on our patient population, the mortality rate was $10.95 \%$, with no significant difference between individuals with and without a refracture. Keene GS et al. reported a 5 -year mortality rate of 56\%, with some sex-related differences [24]. Also, Bliuc D et al. reported a mortality rate attributed to hip fracture compared to populations of similar age, the female sex in $27 \%$, and the male sex in $24 \%$ [22]. The lower refracture rate could explain our lower mortality rate.

\section{Limitation}

The sample size was one of the limitations of our study, which affected finding a statistical significance for our objectives. Another limitation is collecting data from a single center. In addition, considering other risk factors, such as obesity, mobilizing status, and other chronic diseases, may add value to our results. Finally, considering other sites of refracture, such as in the ribs and vertebral fractures, would change the refracture rate in our study.

\section{Conclusions}

Hip fracture is a significant health issue and is an important cause of morbidity and mortality, especially in the elderly population. Our study suggests that there was no association between the refracture rate and osteoporosis, regardless of whether the patient was treated for osteoporosis or not. However, we recommend utilizing a DEXA scan to detect osteoporosis in patients and manage it to improve the outcome of the fracture rate. Moreover, we recommend a systematic review that can include more studies in this field to acquire more definitive results regarding this topic.

\section{Additional Information \\ Disclosures}

Human subjects: Consent was obtained or waived by all participants in this study. King Abdullah International Medical Research Center (KAIMRC) issued approval NRJ21J/085/04. After reviewing your submitted research proposal/protocol and related documents, the IRB has APPROVED the submission . Animal subjects: All authors have confirmed that this study did not involve animal subjects or tissue. Conflicts of interest: In compliance with the ICMJE uniform disclosure form, all authors declare the following: Payment/services info: All authors have declared that no financial support was received from any organization for the submitted work. Financial relationships: All authors have declared that they have no financial relationships at present or within the previous three years with any organizations that might have an interest in the submitted work. Other relationships: All authors have declared that there are no other relationships or activities that could appear to have influenced the submitted work.

\section{Acknowledgements}

This study was supported by King Abdullah International Medical Research Center. We would like to thank Dr. Mohammed Khan for his directions in the statistical analysis and the medical record section of King 


\section{References}

1. Zuckerman JD: Hip fracture. N Engl J Med. 1996, 334:1519-1525. 10.1056/NEJM199606063342307

2. Papaioannou A, Wiktorowicz M, Adachi JD, et al.: Mortality, independence in living, and re-fracture, one year following hip fracture in Canadians. J SOGC. 2000, 22:591-597. 10.1016/S0849-5831(16)31115-6

3. Makridis KG, Karachalios T, Kontogeorgakos VA, Badras LS, Malizos KN: The effect of osteoporotic treatment on the functional outcome, re-fracture rate, quality of life and mortality in patients with hip fractures: a prospective functional and clinical outcome study on 520 patients. Injury. 2015, 46:378-383. 10.1016/j.injury.2014.11.031

4. Kenzora JE, McCarthy RE, Lowell JD, Sledge CB: Hip fracture mortality. Relation to age, treatment, preoperative illness, time of surgery, and complications. Clin Orthop Relat Res. 1984, (186):45-56.

5. Slemenda C: Prevention of hip fractures: risk factor modification . Am J Med. 1997, 103:65-73. 10.1016/S0002-9343(97)90028-0

6. Aspray TJ, Hill TR: Osteoporosis and the ageing skeleton. Subcell Biochem. 2019, 91:453-476. 10.1007/978 981-13-3681-2_16

7. Ma X, Xia H, Wang J, Zhu X, Huang F, Lu L, He L: Re-fracture and correlated risk factors in patients with osteoporotic vertebral fractures. J Bone Miner Metab. 2019, 37:722-728. 10.1007/s00774-018-0974-4

8. Anthamatten A, Parish A: Clinical update on osteoporosis. J Midwifery Womens Health. 2019, 64:265-275. 10.1111/jmwh. 12954

9. Yousefzadeh N, Kashfi K, Jeddi S, Ghasemi A: Ovariectomized rat model of osteoporosis: a practical guide . EXCLI J. 2020, 19:89-107. 10.17179\%2Fexcli2019-1990

10. Ensrud KE, Crandall CJ: Osteoporosis. Ann Intern Med. 2017, 167:ITC17-ITC32. 10.7326/AITC201708010

11. Cram P, Rosenthal GE, Ohsfeldt R, Wallace RB, Schlechte J, Schiff GD: Failure to recognize and act on abnormal test results: the case of screening bone densitometry. Jt Comm J Qual Patient Saf. 2005, 31:90-97. 10.1016/S1553-7250(05)31013-0

12. Lee YK, Ha YC, Choi HJ, Jang S, Park C, Lim YT, Shin CS: Bisphosphonate use and subsequent hip fracture in South Korea. Osteoporos Int. 2013, 24:2887-2892. 10.1007/s00198-013-2395-5

13. Beraldi R, Masi L, Parri S, Partescano R, Brandi ML: The role of the orthopaedic surgeon in the prevention of refracture in patients treated surgically for fragility hip and vertebral fracture. Clin Cases Miner Bone Metab. 2014, 11:31-35.

14. Behanova M, Reichardt B, Stamm TA, Zwerina J, Klaushofer K, Kocijan R: Treatment effects of bisphosphonates and denosumab on survival and refracture from real-world data of hip-fractured patients. Calcif Tissue Int. 2019, 105:630-641. 10.1007/s00223-019-00611-3

15. Murray AW, McQuillan C, Kennon B, Gallacher SJ: Osteoporosis risk assessment and treatment intervention after hip or shoulder fracture: a comparison of two centres in the United Kingdom. Injury. 2005, 36:10801084. 10.1016/i.injury.2005.03.012

16. Davis JC, Guy P, Ashe MC, Liu-Ambrose T, Khan K: HipWatch: osteoporosis investigation and treatment after a hip fracture: a 6-month randomized controlled trial. J Gerontol A Biol Sci Med Sci. 2007, 62:888-891. 10.1093/gerona/62.8.888

17. Saller A, Maggi S, Romanato G, Tonin P, Crepaldi G: Diabetes and osteoporosis. Aging Clin Exp Res. 2008, 20:280-289. 10.1007/BF03324857

18. Geusens PP, van den Bergh JP: Osteoporosis and osteoarthritis: shared mechanisms and epidemiology . Curr Opin Rheumatol. 2016, 28:97-103. 10.1097/BOR.0000000000000256

19. Bultink IE, Lems WF: Osteoarthritis and osteoporosis: what is the overlap? . Curr Rheumatol Rep. 2013, 15:328. 10.1007/s11926-013-0328-0

20. Satomi E, Sitta Mdo C, Machado AN, Garcez Leme LE: Identification and treatment of osteoporosis among elderly patients with hip fractures. Clinics (Sao Paulo). 2009, 64:1201-1204. 10.1590/S1807 59322009001200010

21. Smith MD, Ross W, Ahern MJ: Missing a therapeutic window of opportunity: an audit of patients attending a tertiary teaching hospital with potentially osteoporotic hip and wrist fractures. J Rheumatol. 2001, 28:25042508.

22. Bliuc D, Nguyen ND, Nguyen TV, Eisman JA, Center JR: Compound risk of high mortality following osteoporotic fracture and refracture in elderly women and men. J Bone Miner Res. 2013, 28:2317-2324. 10.1002/jbmr.1968

23. Ryg J, Rejnmark L, Overgaard S, Brixen K, Vestergaard P: Hip fracture patients at risk of second hip fracture: a nationwide population-based cohort study of 169,145 cases during 1977-2001. J Bone Miner Res. 2009, 24:1299-1307. 10.1359/jbmr.090207

24. Keene GS, Parker MJ, Pryor GA: Mortality and morbidity after hip fractures . BMJ. 1993, 307:1248-1250. 10.1136/bmj.307.6914.1248 